\title{
Dietary patterns are associated with biomarkers of chronic low grade systemic inflammation in Scottish postmenopausal women: a prospective analysis
}

\author{
A. D. Wood ${ }^{1}$, W. G. Simpson ${ }^{1,2}$, A. A. Gryka ${ }^{1}$, A. C. Hardcastle ${ }^{1}$, A. Mavroeidi ${ }^{1}$, I. Rothnie ${ }^{2}$, \\ N. J. Vaughan ${ }^{1}$, L. Aucott ${ }^{1}$, G. G. Duthie ${ }^{1}$, F. Thies ${ }^{1}$ and H. M. Macdonald ${ }^{1}$ \\ ${ }^{1}$ University of Aberdeen, Foresterhill, Aberdeen, AB25 2ZD, UK and ${ }^{2}$ Aberdeen Royal Infirmary, Foresterhill, Aberdeen, \\ $A B 252 Z D, U K$
}

Dietary patterns may influence disease risk via effects on chronic low grade systemic inflammation (CLSI). We examined the relationship between dietary patterns and biomarkers of CLSI in Scottish postmenopausal women. Diet was assessed by validated food frequency questionnaire (FFQ) $(n=3238)$ during study visits in 1998-2000, when the mean age [SD] of subjects was 55 [2] years. Dietary patterns were generated by principal components analysis ${ }^{(1)}$. In a recent follow up visit after 11.3 [1.3] years, fasted blood was collected $(n=2039$, $n=1788$ with FFQ data). Serum samples were prepared for analysis of CLSI biomarkers (high sensitivity C-reactive protein (hsCRP), interleukin-6 (IL-6), soluble E selectin (sE selectin), serum amyloid A (SAA)) using standardised immunoassay procedures. ANOVA was used to test the relationship between dietary pattern scores and CLSI biomarker concentrations in a prospective analysis with ANCOVA to control for lifestyle covariates.

Five dietary patterns accounted for $26 \%$ of the variance in the diet ${ }^{(1)}$. Women in the highest quintile of the 'healthy' dietary pattern (rich in fruit, vegetables, lean meat and with low scores for sweet foods) had lower median serum concentrations of hsCRP and IL-6, compared to those in the lowest quintile (Table). These relationships remained significant after adjustment for confounders. Concentrations of hsCRP increased with increasing quintiles of the high fat/white fish dietary pattern; and the dietary pattern with positive factor loadings for bread and butter and negative factor loadings for red meat and alcohol was associated with hsCRP and SAA. These relationships were no longer significant after adjustment for confounding covariates.

A healthy diet rich in fruits, vegetables and lean protein appears to suppress biomarkers of CLSI independently of weight and physical activity. The differences in median serum hsCRP concentration observed between the top and bottom quintiles of healthy diet are clinically relevant, moving subjects from moderate to high risk of future cardiovascular disease ${ }^{(2)}$.

Table. CLSI biomarkers across quintiles of dietary pattern

\begin{tabular}{|c|c|c|c|c|c|c|c|}
\hline \multicolumn{8}{|l|}{ Diet descriptor } \\
\hline 'Healthy' & $Q 1\left(\begin{array}{ll}n & 319)\end{array}\right.$ & $Q 2(n 335)$ & $Q 3(n$ 383) & Q4 (n 366) & $Q 5(n$ 385) & $P^{\mathrm{a}}$ & $P^{\mathrm{b}}$ \\
\hline hsCRP mg/L & $2.10(3.10)$ & $1.80(2.85)$ & $1.60(2.60)$ & $1.50(2.70)$ & $1.50(2.70)$ & 0.017 & 0.03 \\
\hline IL-6 pg/mL & $1.75(1.47)$ & $1.57(1.58)$ & $1.44(1.20)$ & $1.53(1.37)$ & $1.42(1.30)$ & 0.008 & 0.01 \\
\hline $\mathrm{sE}$ selectin $\mathrm{ng} / \mathrm{mL}$ & $38.9(19.2)$ & $37.1(19.9)$ & $36.6(18.2)$ & $37.8(17.2)$ & $36.2(16.9)$ & 0.24 & 0.13 \\
\hline Serum amyloid $\mathrm{A} \mu \mathrm{g} / \mathrm{mL}$ & $39.1(42.0)$ & $38.9(41.5)$ & $34.3(39.3)$ & $34.9(36.4)$ & $35.3(42.3)$ & 0.10 & 0.52 \\
\hline 'High fat \& white fish' & $Q 1(n 390)$ & $Q 2(n 369)$ & Q3 (n 354) & $Q 4(n 355)$ & $Q 5(n 320)$ & $P^{\mathrm{a}}$ & $P^{\mathrm{b}}$ \\
\hline hsCRP mg/L & $1.30(2.40)$ & $1.70(2.78)$ & $1.60(2.85)$ & $1.85(2.98)$ & $2.10(3.20)$ & 0.012 & 0.08 \\
\hline IL-6 pg/mL & $1.38(1.29)$ & $1.50(1.29)$ & $1.45(1.39)$ & $1.65(1.41)$ & $1.63(1.43)$ & 0.07 & 0.23 \\
\hline $\mathrm{sE}$ selectin $\mathrm{ng} / \mathrm{mL}$ & $36.3(17.6)$ & $37.1(16.9)$ & $36.3(18.4)$ & $39.1(19.4)$ & $38.6(20.1)$ & 0.08 & 0.52 \\
\hline Serum amyloid $\mathrm{A} \mu \mathrm{g} / \mathrm{mL}$ & $34.3(38.1)$ & $34.7(38.5)$ & $37.8(44.4)$ & $37.6(42.5)$ & $36.4(39.7)$ & 0.50 & 0.39 \\
\hline 'Bread \& butter, low red meat' & $Q 1(n 351)$ & $Q 2(n 347)$ & $Q 3($ n 378) & Q4 (n 354) & $Q 5(n$ 358) & $P^{\mathrm{a}}$ & $P^{\mathrm{b}}$ \\
\hline hsCRP mg/L & $1.80(3.00)$ & $1.80(2.95)$ & $1.70(2.78)$ & $1.40(2.30)$ & $1.60(2.70)$ & 0.019 & 0.14 \\
\hline IL-6 pg/mL & $1.64(1.40)$ & $1.59(1.42)$ & $1.48(1.35)$ & $1.42(1.22)$ & $1.60(1.36)$ & 0.11 & 0.69 \\
\hline $\mathrm{sE}$ selectin $\mathrm{ng} / \mathrm{mL}$ & $38.6(17.1)$ & $37.6(20.2)$ & $36.0(18.5)$ & $36.1(17.4)$ & $36.8(18.3)$ & 0.07 & 0.38 \\
\hline Serum amyloid $\mathrm{A} \mu \mathrm{g} / \mathrm{mL}$ & $36.4(38.7)$ & $38.0(41.6)$ & $34.9(39.9)$ & $34.8(39.1)$ & $35.9(42.3)$ & 0.02 & 0.23 \\
\hline
\end{tabular}

Data presented as median (IQR) abased on ANOVA with inflammatory marker as the independent variable and dietary pattern quintiles as the fixed factor (unadjusted). ${ }^{\mathrm{b}}$ based on

ANCOVA with adjustment for weight, national deprivation category and physical activity level.

This work was funded by the UK Department of Health. Any views expressed are the authors' own.

1. Hardcastle AC et al. (2011) Eur J Clin Nutr 65, 378-385.

2. Ridker PM et al. (2001) Circulation 103, 1813-1818. 